HNO 2016 $64: 624$

DOI 10.1007/s00106-016-0223-x

Online publiziert: 26 . August 2016

๑) Springer-Verlag Berlin Heidelberg 2016

CrossMark

\title{
T. Hoffmann
}

Klinik für Hals-, Nasen-, Ohrenheilkunde, Kopf- und Halschirurgie, Universitätsklinik Ulm, Ulm, Deutschland

\section{CASH - "Computer-Assisted Surgery around the Head"}

Im aktuellen Heft werden zunächst ge-

Computer begleiten zunehmend unseren (medizinischen) Alltag. Immer mehr Arbeitsschritte werden durch computerisierte Hilfsmittel kontrolliert und unmittelbar beeinflusst. Dies stellt im Operationsbereich eine besondere Herausforderung an die eingesetzte Technik sowie an deren adäquate Bedienung dar.

In der Kopf- und Halschirurgie erfordern die komplexe Anatomie der Schädelbasis und die Enge der assoziierten $\mathrm{Zu}$ gangswege neben kollegialem interdisziplinärem Denken eine hochmoderne i. d. R. computergesteuerte - technische Ausstattung. Unter der Schirmherrschaft der Gesellschaft für Schädelbasischirurgie organisierten die Kliniken für Hals-, Nasen-, Ohrenheilkunde, Mund-Kiefer und Gesichtschirurgie sowie Neurochirurgie der Universität Ulm das interdisziplinäre Symposium „CASH - Computer Assisted Surgery around the Head“.

\section{》) Die Kopf-Hals-Chirurgie erfordert interdisziplinäres Denken und eine hochmoderne technische Ausstattung}

Neben einem klinisch-wissenschaftlichen Austausch wurden die neuesten technischen Entwicklungen präsentiert und praktisch im Rahmen von Handson-Workshops und Livedemonstrationen angewandt. Ausgewählte Beiträge des gut besuchten Symposiums werden nun in 2 Ausgaben aufgeteilt in der Zeitschrift „HNO“ publiziert, um sie einem breiteren Publikum zur Kenntnis $\mathrm{zu}$ bringen. schichtliche Entwicklungen durch die in diesem Zusammenhang bekannten Wegbereiter Ralph Mösges und Marco Caversaccio dargestellt. Der Beitrag von Rainer Wirtz beschreibt die Fortschritte und den Stand der intraoperativen Navigation aus Sicht des Neurochirurgen. Eine Arbeit der Mund-Kiefer- und Gesichtschirurgen Frank Wilde und Alexander Schramm beschäftigt sich mit der Planung und Umsetzung der computerassistierten $\mathrm{Ge}$ sichtsschädelrekonstruktion in der klinischen Routine. Kollegen derselben Fachdisziplin beschreiben zudem die Möglichkeit des Einsatzes der intraoperativen Assistenz zur Qualitätssicherung. Abgeschlossen wird die aktuelle Leitthemenserie mit einer Status-quo-Erhebung und dem Aufzeigen von Zukunftsperspektiven in der Robotik bei Eingriffen in der Kopf-Hals-Chirurgie.

Im Januar 2017 wird im zweiten Heft bei der chirurgischen Simulation u. a. auf ökonomische Aspekte und deren Umsetzung an der Laterobasis eingegangen. Ein weiterer Aspekt wird die Möglichkeit der intraoperativen Bildgebung sein, plastisch ergänzt durch eine Fallseriendarstellung.

Ein primär nichtchirurgischer Teil, aber in der Behandlung von Schädelbasispathologien ebenso wichtiger Beitrag, beschäftigt sich schließlich mit der computergesteuerten Hochpräzisionsbestrahlung im Bereich der Schädelbasis, wobei auf den Begriff der „Radiochirurgie“ in diesem Zusammenhang bewusst verzichtet wurde.

Ich wünsche Ihnen nun eine spannende Lektüre und würde mich im Namen der Mitveranstalter freuen, Sie bei einem Update des Symposiums im Herbst 2017 in Ulm begrüßen zu dürfen.

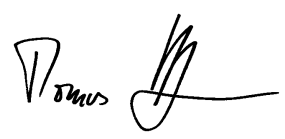

Thomas Hoffmann

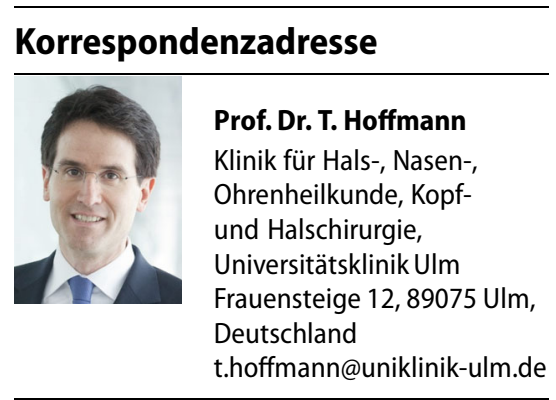

Interessenkonflikt. T. Hoffmann gibt an, dass kein Interessenkonflikt besteht. 